INTELLECTUAL PROPERTY PROTECTION IN VLSI DESIGNS 


\section{Intellectual Property Protection in VLSI Designs}

\section{Theory and Practice}

by

\section{Gang Qu}

University of Maryland, U.S.A.

and

\section{Miodrag Potkonjak}

University of California, Los Angeles, U.S.A. 
eBook ISBN: $\quad 0-306-48717-9$

Print ISBN: $\quad$ 1-4020-7320-8

(C2004 Springer Science + Business Media, Inc.

Print @2003 Kluwer Academic Publishers

Dordrecht

All rights reserved

No part of this eBook may be reproduced or transmitted in any form or by any means, electronic, mechanical, recording, or otherwise, without written consent from the Publisher

Created in the United States of America

Visit Springer's eBookstore at:

http://www.ebooks.kluweronline.com and the Springer Global Website Online at:

http://www.springeronline.com 


\section{Contents}

List of Figures $\quad$ ix

List of Tables $\quad$ xiii

Acknowledgments $\quad$ xix

1. DESIGN SECURITY: FROM THE POINT OF VIEW OF AN EMBEDDED SYSTEM DESIGNER 1

1 Introduction 1

2 Intellectual Property in Reuse-Based Design 2

2.1 The Emergence of Embedded Systems 2

2.2 Intellectual Property Reuse-Based Design 4

2.3 Intellectual Property Misuse and Infringement 8

3 Constraint-Based IP Protection: Examples 9

$\begin{array}{lll}3.1 & \text { Solutions to SAT } & 10\end{array}$

3.2 FPGA Design of DES Benchmark 12

3.3 Graph Coloring and the CF IIR Filter Design 13

4 Constraint-Based IP Protection: Overview 16

4.1 Constraint-Based Watermarking 16

$\begin{array}{lll}4.2 & \text { Fingerprinting } & 17\end{array}$

$\begin{array}{lll}4.3 & \text { Copy Detection } & 18\end{array}$

5 Summary 19

2. PROTECTION OF DATA AND PRIVACY 23

1 Network Security and Privacy Protection 23

2 Watermarking and Fingerprinting for Digital Data 26

3 Software Protection $\quad 29$

4 Summary 31 
3. CONSTRAINT-BASED WATERMARKING FOR VLSI IP PROTECTION

1 Challenges and the Generic Approach 36

1.1 Overview 36

1.2 Watermark Embedding Procedure 37

1.3 Signature Verification Procedure 37

1.4 Credibility of the Approach 38

1.5 Essence of Constraint Addition 39

1.6 Context for Watermarking 40

1.7 Requirements for Effective Watermarks 41

2 Mathematical Foundations for the Constraint-Based Watermarking Techniques 41

2.1 Graph Coloring Problem and Random Graphs $\quad 42$

2.2 Watermarking Technique \#1: Adding Edges 43

2.3 Watermarking Technique \#2: Selecting MIS 47

2.4 Watermarking Technique \#3: Adding New Vertices and Edges $\quad 52$

2.5 Simulation and Experimental Results 53

2.5.1 Numerical Simulation for Techniques \# 1 and \# 2

2.5.2 Experimental Results 54

3 Optimization-Intensive Watermarking Techniques 58

3.1 Motivation 58

3.2 SAT in EDA and SAT Solvers 61

3.3 Watermarking in the Optimization Fashion 63

3.4 Optimization-Intensive Watermarking Techniques for SAT Problem 64

3.4.1 Adding Clauses $\quad 65$

3.4.2 Deleting Literals 66

3.4.3 Push-out and Pull-back 67

3.5 Analysis of the Optimization-Intensive Watermarking Techniques $\quad 69$

3.5.1 The Correctness of the Watermarking Techniques 69

3.5.2 The Objective Function $\quad 70$

3.5.3 Limitations of the Optimization-Intensive Watermarking Techniques on Random SAT $\quad 72$

3.5.4 Copy Detection $\quad 75$

3.6 Experimental Results $\quad 76$

4 Summary 78 
4. FINGERPRINTING FOR IP USER'S RIGHT PROTECTION 81

1 Motivation and Challenges 81

2 Fingerprinting Objectives 83

2.1 A Symmetric Interactive IP Fingerprinting Technique 83

2.2 General Fingerprinting Assumptions 84

2.3 Context for Fingerprinting in IP Protection 85

$2.4 \quad$ Fingerprinting Objectives 85

3 Iterative Fingerprinting Techniques 87

3.1 Iterative Optimization Techniques $\quad 87$

3.2 Generic Approach 88

3.3 VLSI Design Applications 90

3.3.1 Partitioning 91

3.3.2 Standard-Cell Placement 91

3.3.3 Graph Coloring $\quad 92$

$\begin{array}{lll}3.3 .4 & \text { Satisfiability } & 94\end{array}$

$3.4 \quad$ Experimental Results 95

4 Constraint-Based Fingerprinting Techniques 101

4.1 Motivation, New Approach, and Contributions 102

4.2 Generic Constraint-Addition IP Fingerprinting 103

4.3 Solution Creation Techniques 105

4.3.1 Solution post-processing 108

4.4 Solution Distribution Schemes 110

4.5 Experimental Results 111

5 Summary 114

5. COPY DETECTION MECHANISMS FOR IP AUTHENTICATION 117

1 Introduction 117

2 Pattern Matching Based Techniques 119

2.1 Copy Detection in High-Level Synthesis 120

2.2 Copy Detection in Gate-Level Netlist Place-and-Rout 122

2.3 Experimental Results 123

3 Forensic Engineering Techniques 125

$\begin{array}{lll}3.1 & \text { Introduction } & 125\end{array}$

3.2 Forensic Engineering for the Detection of VLSI CAD Tools 126

3.2.1 Generic Approach 126

3.2.2 Statistics Collection for Graph Coloring Problem 128

3.2.3 Statistics Collection for Boolean Satisfiability Problem 131

3.2.4 Algorithm Clustering and Decision Making 132 
3.3 Experimental Results 134

4 Public Detectable Watermarking Techniques 137

4.1 Introduction 137

4.2 Public-Private Watermarking Technique 140

4.2.1 Watermark Selection and Embedding 141

4.2.2 Watermark Detection and Security 142

4.2.3 Example: Graph Partitioning 143

4.3 Theory of Public Watermarking 144

4.3.1 General Approach 144

4.3.2 Public Watermark Holder 145

4.3.3 Public Watermark Embedding 149

4.3.4 Public Watermark Authentication 150

4.3.5 Summary 151

4.4 Validation and Experimental Results 152

4.4.1 FPGA Layout 152

4.4.2 Boolean Satisfiability 153

4.4.3 Graph Coloring 155

5 Summary 157

6. CONCLUSIONS 159

Appendices 163

VSI Alliance White Paper (IPPWP1 1.1) 163

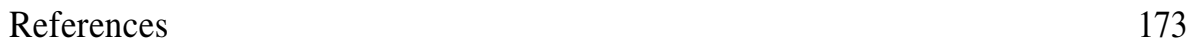




\section{List of Figures}

1.1 Block diagram of the DCAM-103 digital camera (redrawn from the website of LSI Logic Corp.).

1.2 Intellectual property reuse-based design flow. 5

1.3 Design technology innovations and their impact to design productivity.

1.4 A Java GUI for watermarking the Boolean Satisfiability problem.

1.5 Layout of the DES benchmark without watermark(left) and the one with a 4768-bit message embedded (right).

1.6 GUI for watermarking solutions to the graph coloring problem. (top: the greedy 5-color solution to the original graph; middle: a 5-color solution with message UCLA embedded; bottom: a 5-color solution with message VLSI embedded.).

1.7 Design of the 4th order CF IIR filter with watermark. (top: control and datapath of the design implementation; bottom left: control data flow graph; bottom middle: scheduled CDFG; bottom right: colored interval graph.).

1.8 Constraint-based watermarking in system design process, (left: traditional design flow; right: new design flow with watermarking process.).

1.9 Fingerprinting in system design process. (left: iterative fingerprinting technique; right: constraint addition based fingerprinting technique.). 
3.1 Watermark embedding and signature verification process in the constraint-based watermarking method illustrated by the graph coloring problem.

3.2 Key concept behind constraint-based watermarking: additional constraints cut the original solution space and uniqueness of the watermarked solution proves authorship. $\quad 39$

3.3 Pseudo code for technique \# 1: adding edges.

3.4 Example: a graph with message $1998_{10}=11111001110_{2}$ embedded as additional edges.

3.5 Pseudo code for technique \# 2: selecting MIS.

3.6 Example: selecting MISs to embed message $1998_{10}=$ $11111001110_{2}$.

3.7 Numerical simulation data for technique \# 1: the number of edges can be added in with 0 - and 1 -color overhead for random graph $G_{n, 0.5}(500 \leq n \leq 1000)$. The curve in between shows the gain (in terms of the number of extra edges) with one extra color.

3.8 Numerical simulation data for technique \# 2: the number of MISs that can be selected to embed signature with $0-, \quad 1-$, and 2-color overhead for graph $G_{n, 0.5}(500 \leq$ $n \leq 550$ ).

3.9 Coloring the watermarked graph $G_{n, 0.5}$ by technique \# 3: adding new vertex (and its corresponding edges) one by one for $n \in[125,549]$.

3.10 The last 50 instances of graph $G_{n, 0.5}$ in Figure 3.9 $(500 \leq n \leq 549)$.

3.11 An example combinational circuit showing the characteristic function representation.

3.12 Assumptions for decision problem watermarking. 63

3.13 Pseudo code for SAT watermarking: adding clauses. 66

3.14 Pseudo code for SAT watermarking: deleting literals. 66

3.15 SAT watermarking technique: push-out and pull-back. 68

3.16 The satisfiability of model $J(n, r, p)$ (redrawn from [58]). 73

3.17 A SAT instance and its watermarked versions, (a) The initial SAT instance; (b) New instance afteradding clauses;

(c) New instance (same spot as initial) and new curves after deleting literals; (d) New instance after push-out and pull-back.

3.18 Outline of research on constraint-based watermarking. 
4.1 A symmetric interactive fingerprinting IP protection technique. 84

4.2 Basic template for iterative global optimization. 88

4.3 The generic iterative approach for generating fingerprinted solutions.

4.4 Iterative fingerprinting technique in the system design process. 89

4.5 Two-phase fingerprinting technique for IP protection: generating $\mathrm{n}$ solutions and distributing among $\mathrm{m}$ users.

4.6 Solution generation phase of the constraint addition based fingerprinting technique in the system design process.

4.7 Duplicating vertex A to generate various solutions.

4.8 Pseudo code for vertex duplication.

4.9 Manipulating small clique (triangle BCD).

4.10 Constructing bridge between vertices B and E to generate various solutions.

4.11 Choosing a triangle from a graph.

5.1 Pseudo-code for software copy detection at the instruction selection level (pre-processing and detection).

5.2 Example of how RLF and DSATUR algorithms create their solutions. $M D$ - maximal degree; $M S D$ - maximal saturation degree.

5.3 Example of two different graph coloring solutions obtained by two algorithms DSATUR and RLF. The index of each vertex specifies the order in which it is colored according to a particular algorithm.

5.4 Pseudo-code for the algorithm clustering procedure.

5.5 Two different examples of clustering three distinct algorithms. The first clustering (figure on the left) recognizes substantial similarity between algorithms $A_{1}$ and $A_{3}$ and substantial dissimilarity of $A_{2}$ with respect to $A_{1}$ and $A_{3}$. Accordingly, in the second clustering (figure on the right) the algorithm $A_{3}$ is recognized as similar to both algorithms $A_{1}$ and $A_{2}$, which were found to be dissimilar. 
5.6 Each subfigure represents the following comparison (from upper left to bottom right): $(1,3) \pi_{1}$ and NTAB, Rel_SAT, and WalkSAT and $(2,4)$ then zoomed version of the same property with only Rel_SAT, and WalkSAT, $(5,6,7)$ $\pi_{2}$ for NTAB, Rel_SAT, and WalkSAT, and $(8,9,10) \pi_{3}$ for NTAB, Rel_SAT, and WalkSAT respectively. The last five subfigures depict the histograms of property value distribution for the following pairs of algorithms and properties: (11) DSATUR with backtracking vs. maxis and $\pi_{3},(12)$ DSATUR with backtracking vs. tabu search and $\pi_{7},(13,14)$ iterative greedy vs. maxis and $\pi_{1}$ and $\pi_{4}$, and (15) maxis vs. tabu and $\pi_{1}$.

5.7 Constructing public-private watermark messages.

5.8 Public watermark on graph partitioning problem. (a) The original graph partitioning instance; (b) the same graph with 8 marked pairs that enables an 8-bit keyless public watermark; (c) A solution with public information "01001111"; and (d) A solution with public information "01110000".

5.9 General approach of the public watermarking technique. 145

5.10 Creating keyless public watermark from public signature. 150

5.11 Four instances of the same function with fixed interfaces (redrawn from [97]).

5.12 Hamming distance among the four public watermark messages. The bottom half comes from the message header(plain text part), and the top half comes from the message body(results of RC4).

5.13 Four GC solutions with different public watermarks added to the same graph. 


\section{List of Tables}

3.1 MISs selection step-by-step: build the first MIS by selecting vertices one-by-one according to the embed message, reorder the remaining vertices, and build the second MIS.

3.2 Coloring the watermarked random graph $G_{n, 0.5}$ : (i) adding $n$ edges; (ii) adding $2 n$ edges; (iii) selecting one MIS ( $n=125,250,500,1000)$.

3.3 Coloring the watermarked dense/sparse graph $G_{n, p}$ for $n=125,250,500$ and $p=0.1,0.9$.

3.4 Coloring the watermarked DIMACS benchmark.

3.5 Coloring the watermarked real-life graphs by: (i) adding $n$ edges; (ii) selecting one MIS; (iii) adding one new vertex. IVI: number of vertices; |El: number of edges; k: minimal number of colors.

3.6 Characteristic functions for simple gates[100].

3.7 Characteristics of benchmarks. "Ratio" is measured by literals/clauses and "Clause Length" is the range for the length of clauses.

3.8 Improvement of the optimization-intensive technique over regular watermarking technique.

4.1 Test cases for partitioning experiments.

4.2 Results for the fingerprinting flow on three standard bipartitioning test cases. Tests were run using actual cell areas, and a partition area balance tolerance of $10 \%$. Each trial consists of generating an initial solution, then generating a sequence of 20 fingerprinted solutions. All results are averages over 20 independent trials. 
4.3 Test cases for standard-cell placement experiment.

4.4 Standard-cell placement fingerprinting results for the Test2 instance. We report CPU time (mm:ss) needed to generate each solution, as well as total wirelength costs normalized to the cost of the initial solution $S_{0}$. Manhattan distances from $S_{0}$ are given in $10^{6}$ microns.

4.5 Summary of results for fingerprinting of all four standardcell placement instances. "Original" lines refer to the initial solutions $S_{0}$. All other lines refer to fingerprinted solutions $S_{i}, i>0$. Manhattan distance is again expressed in $10^{6}$ microns.

4.6 Results for coloring the DIMACS challenge graph with iterative fingerprinting.

4.7 Number of undetermined variables (Var.), average distance from original solution (Distance), and average CPU time (in $1 / 100^{\text {th }} \mathrm{s}$ of a second) for fingerprinting SAT benchmarks.

4.8 Summary of the four fingerprinting techniques. 109

4.9 Characteristics of benchmark graphs from real life. 112

4.10 Coloring the fingerprinted graph DSJC1000.5.col.b. 112

4.11 Coloring the fingerprinted real-life benchmark graphs.

5.1 Effectiveness of the copy detection mechanism for behavioral specifications.

5.2 Matching percentage between two full designs, based on weighted sum of credits. The matching percentage between Cases $\mathrm{E}$ and $\mathrm{F}$ may be high because of potential reused IP between these designs.

5.3 Percentage of matching between partial design and full design with weighted sum of the credits. Each entry is an average over three experimental trials.

5.4 Experimental Results: Graph Coloring. A thousand test cases were used. Statistics for each solver were established. The thousand instances were then classified using these statistics.

5.5 Experimental Results: Boolean Satisfiability A thousand test cases were used. A thousand test cases were used. Statistics for each solver were established. The thousand instances were then classified using these statistics. 
5.6 Average number of different bits in public message body ("body"), average distance (rounded to integer) from the original solution ("sol.") when 4-bit, 8-bit, 16-bit, and 32-bit forgery is conducted to the public message header on SAT benchmarks.

5.7 Embedding public watermark to real-life graphs and randomized graphs.

A.1 Example Security Schemes Applicable During VC LifeCycle: $\mathrm{D}=$ Development, $\mathrm{L}=$ Licensing, $\mathrm{I}=\mathrm{VC}$ Integration, $\mathrm{M}=$ Manufacture, $\mathrm{U}=$ End Component Use, $\mathrm{A}$ = End Application, ID = Infringement Discovery.

A.2 Example VC Protection Scheme Summary: LA = Legal Agreement, DF= Digital Fingerprint, DW= Digital Watermark, E= Encryption, F= Antifuse FPGA. 
To my parents, my wife, and my son.

-Gang $Q u$ 


\section{Acknowledgments}

Intellectual property protection of hardware and software artifacts is of crucial importance for a number of dominating business models. Maybe even more importantly, it is an elegant and challenging scientific and engineering challenge. This book provides in detailed treatment of our newly developed constraint-based protection paradigm for the protection of intellectual properties in VLSI CAD. The key idea is to superimpose additional constraints that correspond to an encrypted signature of the designer to design/software in such a way that quality of design is only nominally impacted, while strong proof of authorship is guaranteed. Its basis is the Ph.D. dissertation of the first author. In addition, it also presents a few of the most recent research results from both authors and their colleagues.

We are grateful to our co-authors who greatly contributed to research presented in this book including Andrew Caldwell, Hyun-Jin Choi, Andrew Kahng, Darko Kirovski, David Liu, Stefanus Mantik, and Jennifer Wong. In addition, we would also like to thank a number of other researchers, including Jason Cong, Inki Hong, Yean-Yow Huang, John Lach, William Magione-Smith, Igor Markov, Huijuan Wang, and Greg Wolf for numerous advises and even more numerous helpful discussions.

We would also like to acknowledge Virtual Socket Interface Alliance for allowing us to include its document, "Intellectual Property Protection White Paper: Schemes, Alternatives and Discussion Version 1.1", as the appendix. Special thanks to Stan Baker, Executive Director of VSI Alliance, and Ian Mackintosh, author of the above document, for making this happen.

Finally, we would like to thank Pushkin Pari and Jennifer Wong for careful reading of the manuscript and for providing us invaluable feedback. We would like to express appreciation to our publishing editor, Mark de Jongh, for his help throughout this project. Any errors that remain are, of course, our own.

Gang Qu

College Park, Maryland

gangqu@glue.umd.edu
Miodrag Potkonjak

Los Angeles, California

miodrag@cs.ucla.edu

September 2002 